\title{
Characterization, Micellization Behavior and Performance of a Novel Surfactant Derived from Gundelia Tournefortii Plant during Chemical EOR
}

\author{
Hossein Bahraminejad ${ }^{1}$, Abbas Khaksar Manshad ${ }^{1}$, Alireza Keshavarz ${ }^{2}$ \\ ${ }^{1}$ Department of Petroleum Engineering, Abadan Institute of Technology, Petroleum University of Technology \\ (PUT), Abadan, Iran. \\ ${ }^{2}$ School of Engineering, Edith Cowan University, Joondalup, WA 6027, Australia
}

\section{Supplementary Information:}

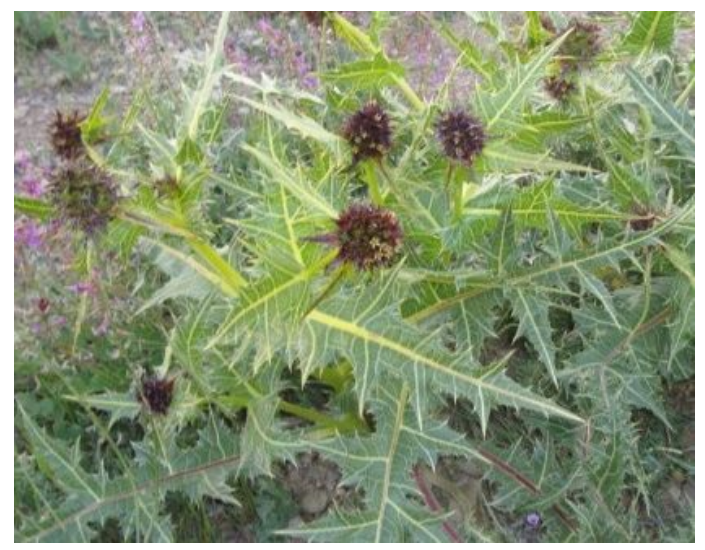

Figure S1: The Gundelia tournefortii plant.

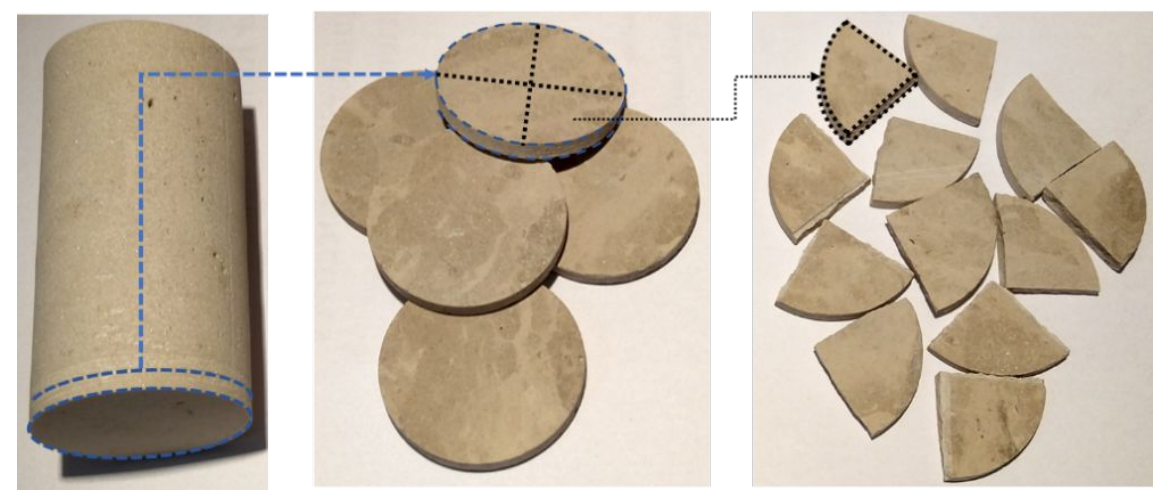

Figure S2: Procedure of pellet preparation from carbonate rock sample. 
Table S1. Carbonate rock sample plug characterization

\begin{tabular}{|c|c|c|}
\hline Parameter & Value & Description of calculations \\
\hline Diameter $(\mathrm{cm})$ & 3.7 & Measurement of D by a Vernier scale \\
\hline Length $(\mathrm{cm})$ & 5 & Measurement of L by a Vernier scale \\
\hline Bulk volume $\left(\mathrm{cm}^{3}\right)$ & 53.73 & $B V=\frac{\pi D^{2}}{4} L$ \\
\hline Pore volume $\left(\mathrm{cm}^{3}\right)$ & 6.44 & $\begin{array}{c}P V= \\
\frac{(F B \text { saturated weight of core plug-Dry weight of core plug) }}{F B \text { density }}\end{array}$ \\
\hline Porosity (\%) & 12 & $\Phi=\frac{P V}{B V}$ \\
\hline Permeability (md) & 5 & $\begin{array}{l}\qquad K=K g-c\left(\frac{1}{P_{m}}\right) \\
\text { The first term is gas permeability and the second term is the Klinkenberg } \\
\text { effect correction }\end{array}$ \\
\hline OOIP $\left(\mathrm{cm}^{3}\right)$ & 4.44 & $\begin{array}{c}\text { Step 1: Saturation of core plug with FB } \\
\text { Step 2: Flooding crude oil into the FB saturated core plug until the outlet } \\
\text { fluid shows } 100 \% \text { crude oil and } 0 \% \text { FB } \\
\text { Step 3: Measurement of total evacuated FB } \\
\text { OOIP = Total evacuated FB }\end{array}$ \\
\hline $\begin{array}{l}\text { Irreducible water } \\
\text { saturation }(\%)\end{array}$ & 31 & $\mathrm{~S}_{\mathrm{wirr}}=\mathrm{PV}-$ Original Oil in Place (OOIP) \\
\hline
\end{tabular}


Table S2. Percentages of the components of the light dead crude oil used in this study.

Component 
Table S3. Formation brine properties.

\begin{tabular}{cccccccc}
\hline Component & Sodium & Calcium & Magnesium & chloride & sulfate & Bicarbonate & TDS \\
\hline $\begin{array}{c}\text { Concentration } \\
(\mathbf{p p m})\end{array}$ & 22,356 & 5,200 & 1,400 & 34,506 & 95 & 67 & $\mathbf{6 2 , 0 0 0}$ \\
\hline
\end{tabular}


Table S4. Seawater compositional analysis.

\begin{tabular}{ccccccccc}
\hline Component & Sodium & Calcium & Magnesium & Chloride & Sulfate & Potassium & TDS & PH \\
\hline $\begin{array}{c}\text { Concentration } \\
\text { (ppm) }\end{array}$ & 7337 & 1920 & 936 & 11340 & 6892.8 & 92.43 & 33194 & 7.67 \\
\hline
\end{tabular}

